\title{
GPRS Based LAN Monitoring and Controlling
}

\author{
${ }^{1}$ Meghana Sapkal, ${ }^{2}$ Shekhar Patil, ${ }^{3}$ Leesensa Vispute, ${ }^{4}$ Santosh Jagtap. \\ Guided by Prof. S.C. Chaudhari \\ ${ }_{1,2,3,4}$ Department of Information Technology, \\ Padmabhooshan Vasantdada Patil Institute of Technology, \\ University of Pune, India
}

\begin{abstract}
The GPRS based LAN monitoring and controlling system is mainly developed to focus on expansion of various networks services or facilities which are essential for brilliantly monitoring a LAN network. In this system, workstations are collected to arrange them in a network. Managing and regulating the activities in the network while at the workplace is an easy task. But, while you are outstation it becomes difficult to monitor and control. You can always have your smart handset or GPRS enable device of any type which will fulfill this purpose. The interface between the clients and the remote administrator is achieved via the central monitoring server through sending and receiving email only. The project targets to develop an integrated software solution also this permits a network supervisor to distantly monitor the LAN. This is done by his email compatible devices and can see tasks on the client side. This project is developed for giving out the best particulars about a network to its administrator, on his GPRS enable device, when he is at a remote location.
\end{abstract}

Keywords: LAN, GSM, GPRS, Remote Monitoring \& Control, Desktop.

\section{Introduction}

"GPRS based LAN monitoring and controlling" is developed to regulate and monitor the LAN network from our wireless handheld device, i.e. a smart phone using email at anyplace deprived of limitation of distance. You have a LAN setup at your agency or bureau. By sitting at house if you want to recognize the standing of LAN then you can do it by just sending an email. Wireless devices are broadly used and it has breached every part of our life. To distantly monitor a network through email system is a mirage, this concept is an attempt to get to this mirage a reality, and this is where the genesis of this concept lays LAN monitoring using GPRS technology can be employed in administrative bureau, shopping mall as well as academy or academy level.

The main objective of GPRS based LAN monitoring and controlling is to provide maximum details about the network to the administrator on their smart phone, when the administrator is away from the office goes out the station. Every time when administrator goes away from the server, it can be more dangerous as there is no control of anyone over the server. It may convert into any bad mishap like client will not do their allocated work, clients can be idle without doing any work, and the client can access unauthorized files or can access restricted sites over the Internet.

In the organization the overseer is responsible for turnover or damage of the representation. It is more vital that the overseer will have to be persistently in touch with the server and it results in value-added efficiency of the enterprise. When it is required to release a new version of the software, it is helpful to take reference to previous versions.

If designers identify in advance which approaches of software may be reformed or will cause errors, then developers can focus more on these faults. This will result in evolving, innovative software.

\section{Related Work}

This part clarifies definite fundamentals of GSM built LAN observing in which SMS leftovers the greatest effective communication system for advancing the content onto the cell phone devices. This is a server based application that provides in which there is the ability to Send and receive SMS messages. The network used here is GSM network, which requires TCP/IP protocol. 


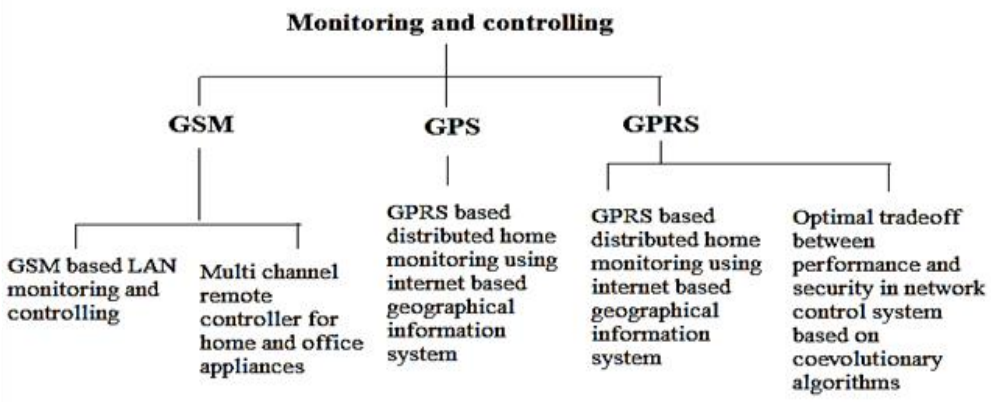

Figure.1- Classification of Monitoring and Controlling

\subsection{GSM Based Monitoring and Controlling:-}

\subsubsection{A Multi-Channel Remote Controller for Home and Office Appliances}

Hamit Erdem and Armagan ner," A Multi-Channel Remote Controller For Home and Office Appliances ", IEEE Transactions on Consumer Electronics, Vol. 55, No. 4, November 2009.

This paper introduces the conception and execution of a multi-channel remote controller for family and bureau applications. The core impression behind this effort is to combine selected present remote controller stations in a mutual platform. The suggestion is that the controller is more trustworthy than conventional ones, especially during an emergency condition such as main server failure and interruption in GSM. The recommended system allows supple entrée to controlled devices. [1]

\subsubsection{GSM Based LAN Monitoring System}

Amol Poman, Mahesh Gundras, Prashant Pujari Department Of Computer Engineering GHRCEM, University of Pune," GSM Based LAN Monitoring System", Amol Poman et al, / (IJCSIT) International Journal of Computer Science and Information Technologies, Vol. 3 (3) , 2012 ,3848-3851.

The phone-based interface would relay the commands to a server system that would perform the essential action and return a purpose completion SMS that would be sent to the administrator. This suggested that developed system has provided a low cost, secure, and accessible, remotely monitored and controlled solution for LAN monitoring using GSM. [2]

\subsubsection{GSM Based LAN Monitoring and Controlling}

Mamata Bhamare, Tejashree Malshikare, Renuka Salunke, Priyanka Waghmare Department of computer engineering, MIT pune-411038, India, "GSM Based LAN Monitoring and Controlling ", International Journal of Modern Engineering Research (IJMER) Vol.2, Issue.2, Mar-Apr 2012 pp-387-389.

The project aims to develop various network utilities which are required to effectively monitor a LAN network. It aims to build up an integrated software solution that allows a network administrator to remotely monitor his LAN by his cell phone. This project is to offer the most details about the network to the administrator on their mobile phone, when administrator when is away from office or goes out of station. [3]

\subsection{GPS Based Monitoring and Controlling:-}

\subsubsection{GRPS-Based Distributed Home-Monitoring Using Internet-Based Geographical Information System}

A.R. Al-Ali, Imran A. Zualkernan, Assia Lasfer, Alaa Chreide, and Hadel Abu Ouda," GRPS-Based Distributed Home-Monitoring Using Internet-Based Geographical Information System" , IEEE Transactions on Consumer Electronics, Vol. 57, No. 4, November 2011.

It presented the publicly available web based Geographical Information Systems (GIS) and the accessibility of low-cost integrated General Packet Radio Service (GPRS) /Global Positioning Systems (GPS) modem has enabled the evolution of embedded stand-alone home monitoring systems. Local range monitoring systems have been extended to a wider remote range using GSM/GPRS networks and wireless TCP/IP based communications.[4] 


\subsection{GPRS Based Monitoring and Controlling:-}

\subsubsection{Optimal Tradeoff between Performance and Security in Networked Control Systems Based on Co evolutionary Algorithms}

Wente Zeng, Student Member, IEEE, and Mo-Yuen Chow, Fellow, IEEE," Optimal Tradeoff between Performance and Security in Networked Control Systems Based on Co evolutionary Algorithms ", IEEE transactions on industrial electronics, vol. 59, no. 7, July 2012.

This theme gives a theoretical account of public presentation, security tradeoff optimization based on a Co evolutionary genetic algorithm (CGA) for the networked DC motor system. Experiments are described that CGA is very accomplished in finding the Nash equilibrium for the tradeoff model on the NCS.[5]

\subsubsection{Remote computer access through Android mobiles}

Jaya Bharathi chintalapati, Srinivasa Rao T.Y.S Department of Computer Science Engineering," Remote computer access through Android mobiles", IJCSI International Journal of Computer Science Issues, Vol. 9, Issue 5, No 3, September 2012.

In this paper, they have presented the process to access the computers with the help of android mobile phones. This process is based on VNC (virtual network computing). The image of the desktop is compressed before it is transmitted to the cellular phone. There are numerous functions delivered so as to ease the viewing on smart phones. Shortcut functions can be used to quickly access the often used area. Existing key assignments can be viewed using guidance function. This organization has provided mobility for users for checking their computer desktops over the net. More facilities and features for accessing applications running on remote desktop from mobile handheld devices will be provided. Thus the extended scope of this system will prove to be helpful in providing mobility and accessing the remote desktop over the internet. [6]

\subsubsection{Virtual Network Computing Viewer Using Remote Frame Buffer Protocol}

Nandhini. S, Archana. N, Bagavathi. S, Arunachalam. M 1,2,3,4 Department of Computer Science and Engineering, "Virtual Network Computing Viewer Using Remote Frame Buffer Protocol ", International Journal of Engineering and Innovative Technology (IJEIT) Volume 2, Issue 8, February 2013.

Virtual Network Computing (VNC) is a remote control system which allows the user to view and interact with one computer (the server) to another computer or cellular phones (the viewer or client) anywhere on the internet. The system to be accessed must be running a VNC server and it must be attached to a network. A proxy is used for sending the copy of the desktop to the phone, to convert different devices, to overpower network traffics. VNC is a simple protocol for remote access to graphical user interfaces. [7]

\section{The Proposed Approach}

You can see the components below. Only one server is there, but several clients are there and the email system. These three things are the main parts of the system. Here, when the overseer is beyond the locality, he can log into the valid email account. Then he can monitor and control the LAN.

When the administrator log into the authenticated account and then he can use the services as net view, client list, shut down, restrictions to the access, kill process, open file, delete file, etc. By using the facilities provided by POP3 (Post Office Protocol) server. Then over seer can communicate with the server machine and get expected results.

We are using one technique used which is called as a message parser and by using this technique the server machine can recognize the commands send by the administrator and vice versa. It will allocate the resources to the client demanded by a particular client. It will give a clear view to the administrator and makes work simple.

The Overseer sends request to the server through email. After recognition of the client by the overseer, he is then able to monitor and extract data from the data buffer. In which latest $15 \mathrm{Sec}$ data onto each machine is updated or kept and sends this info to the overseer as a response.

The Administrator is provided with a GUI based application in J2ME for sending command messages instantly without the need to retype messages all the time. The Server sends commands to the clients in net.

Communication is done with the GPRS enable device in which client connects with the server and the server connects with the client. All clients are controlled as well as monitored by the administrator via a series of emails.

The administrator controls the LAN through his GPRS enable device even if he is in the distant place. The clients communicate with the administrator. LAN monitoring and controlling using GPRS technology can be used in various things like in administrations, shopping center as well as institutional or university level. 


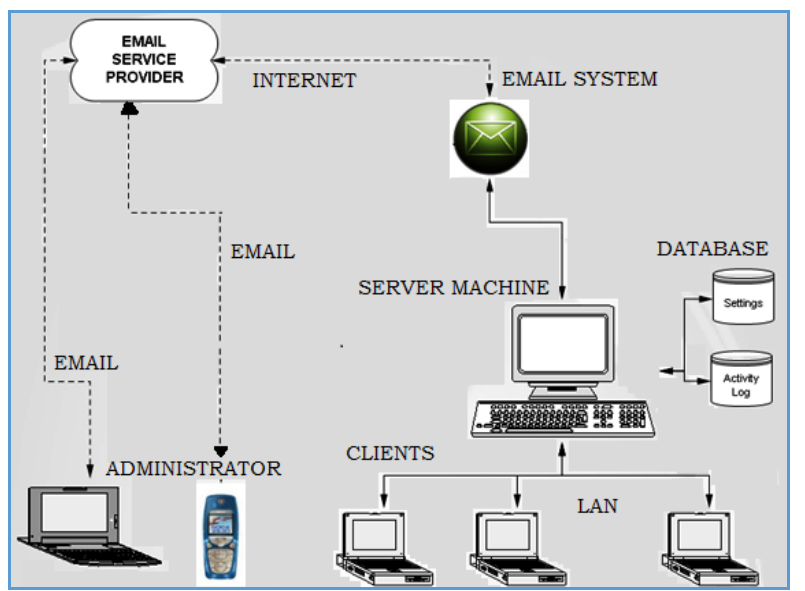

Figure. 2 - Architecture of LAN monitoring and controlling.

Here, GPRS enabled device is sending emails to the host. In the email, there is a dispatcher's Name. The name is then cross-checked with the names in a database. The subject, i.e. which email is sent to the host. The server recognizes the client from list of clients.

By using parser we identify the insides of an email. By using process builder class we perform this process for a special client. Then after the achievement of a demanded action on the client, the client sends the response to the server. Then the server responds to the administrator through email again parsing is used to send email to administrator specify that operations on the client is performed.

In that respect is no any database maintained in this project, there is only one database, it can be a file i.e. any type of secondary storage. Through the database, we get the data as we want.

\subsection{Work Flow}
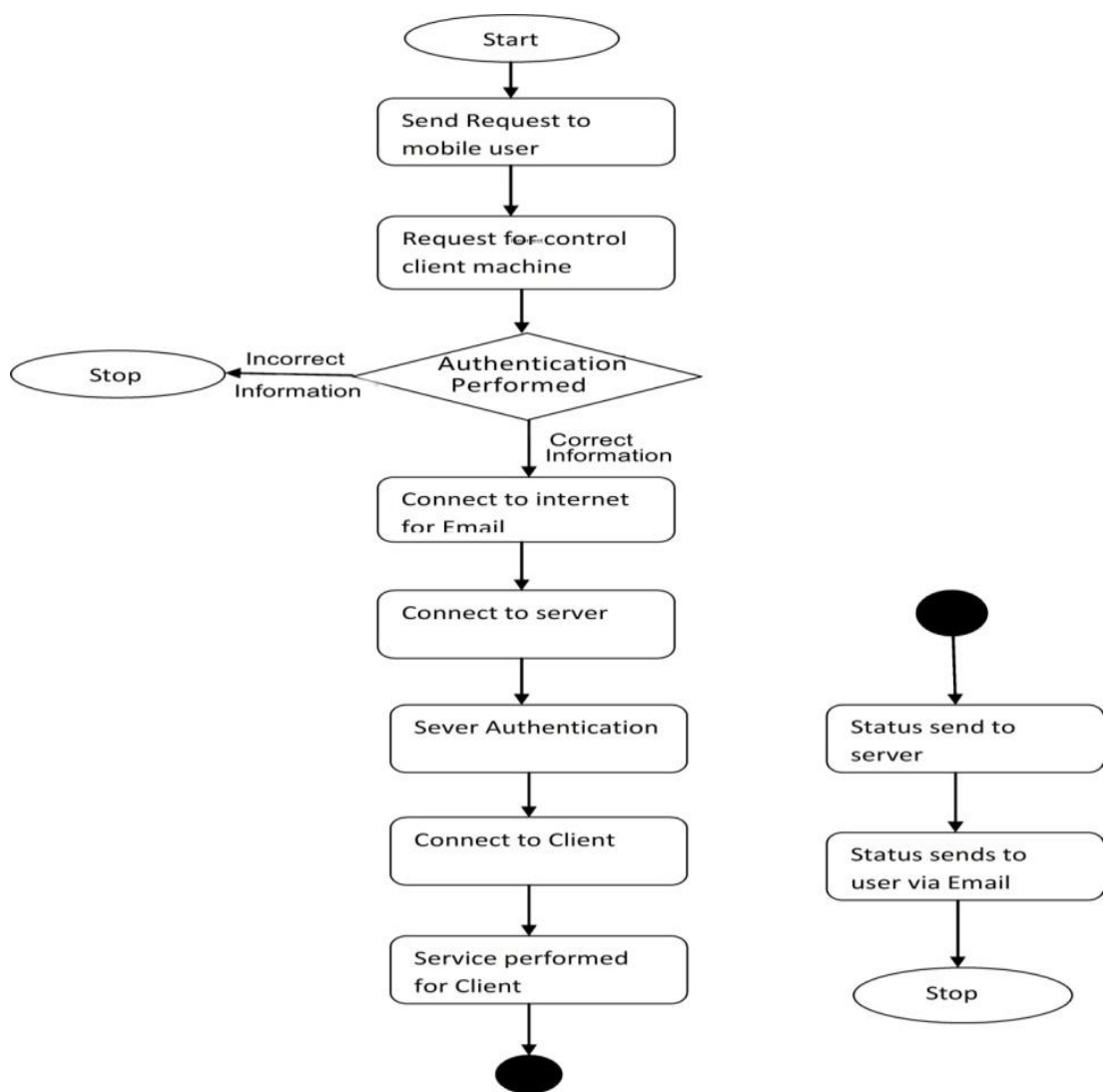

Figure.3- Workflow of Proposed System 


\subsection{Project Work:}

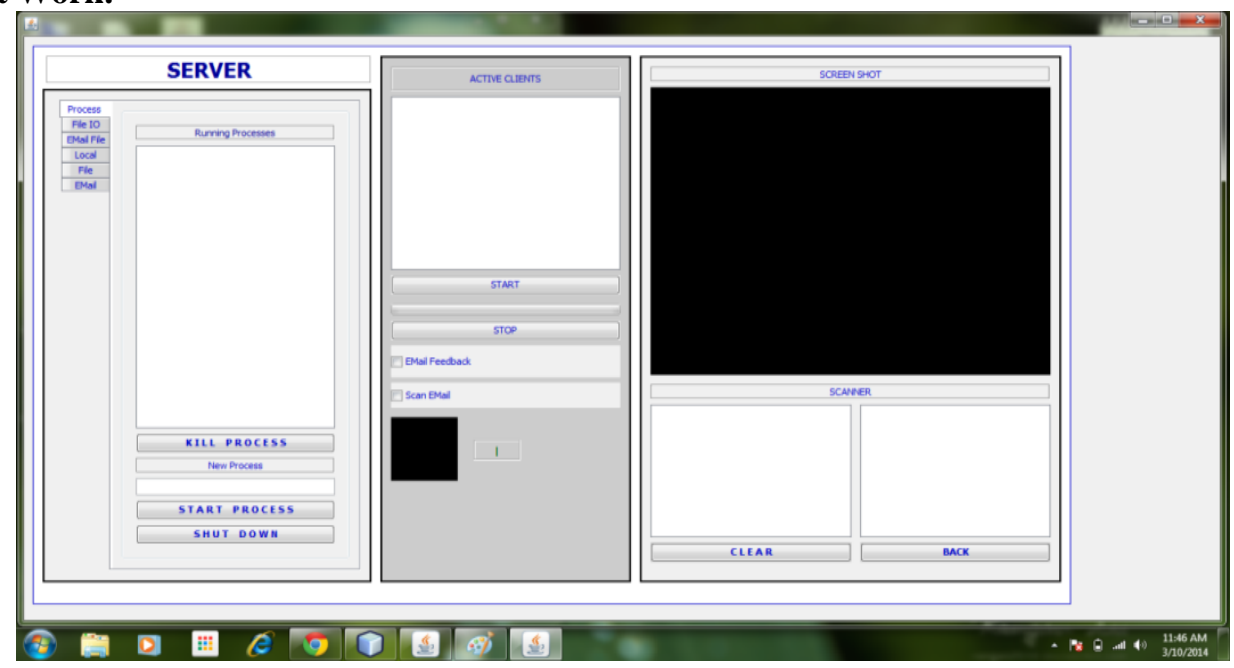

Figure.4- Server module without starting the server

Here is the main server module screen shot. This is the screen shot when server module is off. This will give a clearer idea about what are our actual module and their facilities etc.
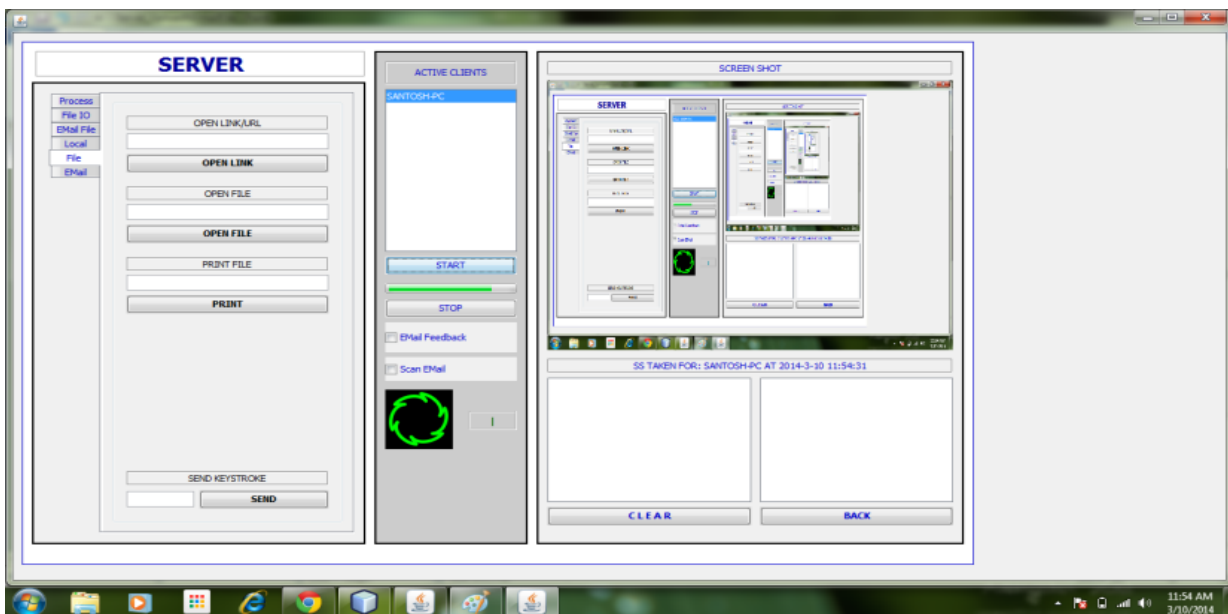

Figure.5- Server module while it is active.

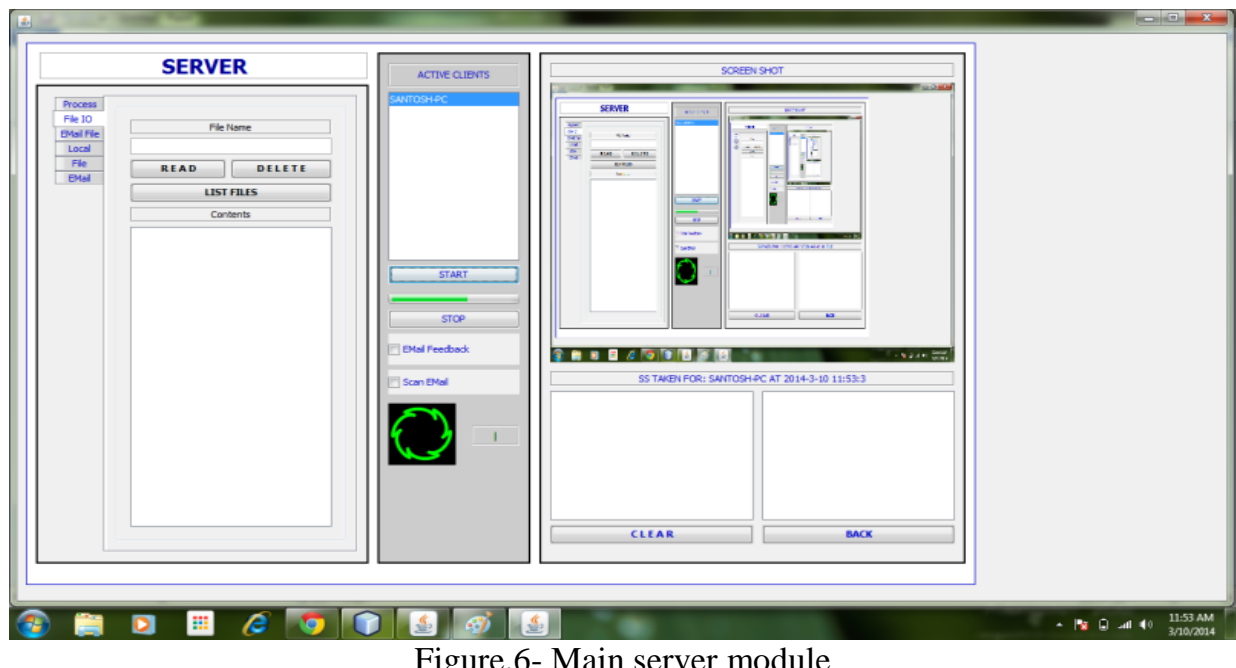

Figure.6- Main server module

This is a screenshot of our main server module. It will give the detail knowledge idea about the LAN. The list of active clients and their status etc. We are providing the services like kill process, new process, open 
file, closing the file with their desktop (screen's) shot and providing the email fetcher and receiver. There is one list present of active clients. We have to select the client then their related services can use by us.

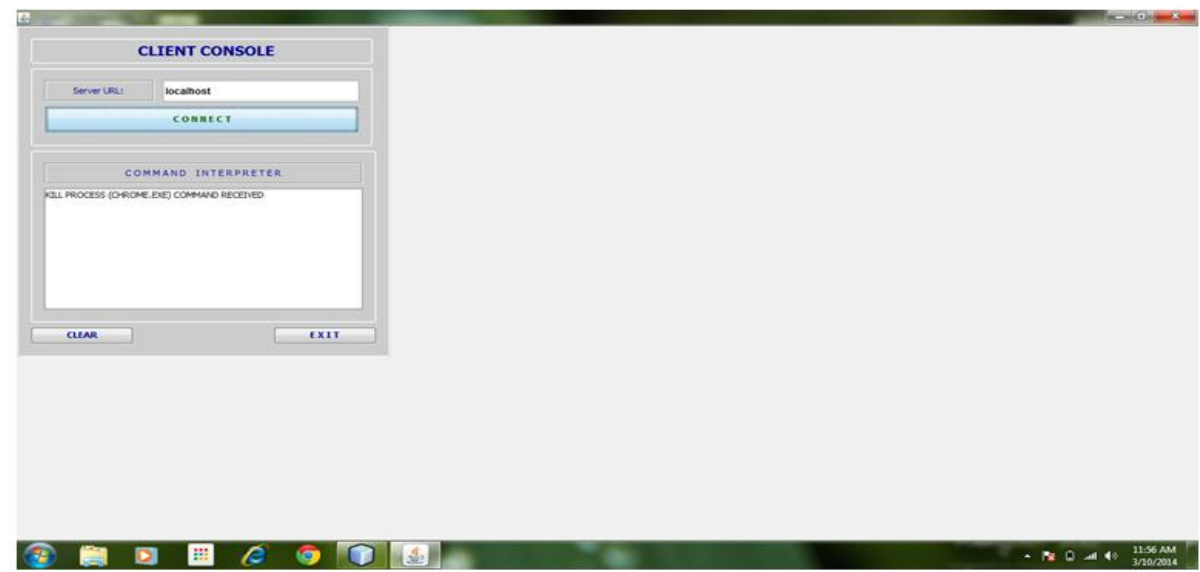

Figure.7- Client side module

Here is the client side module of our project which is used to connect the client to server module and use the facility provided by server like opening files, using the resources etc. Here we have to enter the IP address of the client machine and click the connect button to connect. The connect button will use the programming structure and connect to that machine to the server module.

\section{Conclusion}

This paper explains the basics of GPRS based LAN monitoring and controlling system. Email remains the most proficient communication system for pushing the content on to the devices because every professional worker uses the electronic message scheme. The system will provide a low price, protected, manageable, remotely monitored and controlled result for LAN monitoring using GPRS technology. Demon tool of our scheme will manage the joining between the clients and server of our system which will manage the services and control it. When any emails related to any command comes at email fetcher, it will automatically read and consider the appropriate action by server module. The server module will remain active on a server and will offer the power to post and receive Email messages through GPRS network and communicates through the standard SMTP/POP3 protocol. The host module is responsible for all the services which offered to the guest.

\section{Acknowledgements}

First and foremost, we would like to thank our project guide Prof S. C. Chaudhari and Head of the Department Prof. Vaishali. S. Nandedkar for the valuable guidance. Their willingness to motive us contributed greatly to our project. Besides, we would also like to thank our Principal Dr. Y. V. Chavan, all the staffs and authorities of the Padmabhooshan Vasantdada Patil Institute of Technology, University of Pune for providing us with good environment and facilities to complete our project.

\section{References}

[1]. HamitErdem and Armaganner," A Multi-Channel Remote Controller For Home and Office Appliances ", IEEE Transactions on Consumer Electronics, Vol. 55, No. 4, November 2009.

[2]. Amol Poman, Mahesh Gundras, Prashant Pujari Department Of Computer Engineering GHRCEM, University of Pune," GSM Based LAN Monitoring System", Amol Poman et al, / (IJCSIT) International Journal of Computer Science and Information Technologies, Vol. 3 (3) , 2012 ,3848-3851.

[3]. Mamata Bhamare, Tejashree Malshikare, Renuka Salunke, Priyanka Waghmare Department of computer engineering, MIT pune411038, India, "GSM Based LAN Monitoring and Controlling ", International Journal of Modern Engineering Research (IJMER) Vol.2, Issue.2, Mar-Apr 2012 pp-387-389

[4]. A. R. Al-Ali, Imran A. Zualkernan, Assia Lasfer, Alaa Chreide, and Hadel Abu Ouda," GRPS-Based Distributed Home-Monitoring Using Internet-Based Geographical Information System" , IEEE Transactions on Consumer Electronics,vol.57 ,no 4,November 2011.

[5]. Wente Zeng, Student Member, IEEE, and Mo-Yuen Chow, Fellow, IEEE," Optimal Trade-off Between Performance and Security in Networked Control Systems Based on Co evolutionary Algorithms ", IEEE transactions on industrial electronics, vol. 59, no. 7, July 2012 .

[6]. Jaya Bharathi chintalapati, Srinivasa Rao T.Y.S Department of Computer Science Engineering," Remote computer access through Android mobiles", IJCSI International Journal of Computer Science Issues, Vol. 9, Issue 5, No 3, September 2012.

[7]. Nandhini. S, Archana. N, Bagavathi. S, Arunachalam. M 1,2,3,4 Department of Computer Science and Engineering, "Virtual Network Computing Viewer Using Remote Frame Buffer Protocol ", International Journal of Engineering and Innovative Technology (IJEIT) Volume 2, Issue 8, February 2013 
[8]. Archana Jadhav, Vipul Oswal, Sagar madne, Harshal zope, vishal hatmode Dept. of Information Technology RSCOE, Pune411033, India, "VNC architecture based remote desktop access through android mobile phone" International Journal of Advanced Research in Computer and Communication Engineering Vol. 1, Issue 2, April 2012

[9]. Vladimir V. Riabov, Rivier College "SMTP (Simple Mail Transfer Protocol)" JWBS001A-60.tex WL041/Bidgoli WL041Bidgoli.cls May 12, 2005

[10]. David Reilly, Michael Reilly," Java ${ }^{\mathrm{TM}}$ Network Programming and Distributed Computing” Publisher: Addison Wesley, Pub Date March 25, 2002 ISBN: 0-201-71037-4 